\title{
Date of Last Administration
}

National Cancer Institute

\section{Source}

National Cancer Institute. Date of Last Administration. NCI Thesaurus. Code C156819.

The date of the last administration of the substance of interest. 